Historic, Archive Document

Do not assume content reflects current scientific knowledge, policies, or practices. 



\title{
Gillett's Fern and Flower Farm
}

\author{
KENNETH E. GILLETT, Proprietor
}

Southwick, - Massachusetts

Orders accepted under usual trade terms and conditions, boxing at cost.

The following stock I have in access of requirements for my usual retail trade and am pleased to make these offerings to the trade.

All of the stock is of my own growing and is now in my nursery ready for shipment.

This list supersedes all previous catalogs and quotations.

Thirty of one variety will be sold at the hundred rate; five at the ten rate; where less than five of one variety are ordered add $25 \%$ to the ten rate.

\section{HARDY NATIVE FERNS}

\begin{tabular}{|c|c|c|c|}
\hline & & Per 10 & Per 100 \\
\hline 7000 & Adiantum pedatum (Maidenhair Fern) & $\$ 1.00$ & $\$ 8.00$ \\
\hline 15000 & Aspidium acrostichoides (Christmas Fern) & 1.00 & 8.00 \\
\hline 1000 & Aspidium aculeatum Braunii (Prickly Shield Fern) & 2.30 & 20.00 \\
\hline 900 & Aspidium cristatum (Crested Wood Fern) & 1.00 & 8.00 \\
\hline 1200 & Aspidium cristatum Clintonianum & 1.00 & 8.00 \\
\hline 500 & Aspidium Filix-mas (Male Fern) & 2.30 & 20.00 \\
\hline 1500 & Aspidium Goldieanum (Goldie's Wood Fern) & 3.00 & 25.00 \\
\hline 5000 & Aspidium marginale (Evergreen Wood Fern) & 1.00 & 8.00 \\
\hline 300 & Aspidium munitum (Giant Holly Fern) & 2.00 & 18.00 \\
\hline 1000 & Aspidium noveboracense (New York Shield Fern) & 1.00 & 8.00 \\
\hline 6000 & Aspidium spinulosum (Spinulose Wood Herm) & 1.00 & 8.00 \\
\hline 1000 & Aspidium spinulosum dilatatum & 1.35 & 10.00 \\
\hline 500 & Aspidium thelypteris (Marsh Shield Fern) & 1.00 & 8.00 \\
\hline 0 & Asplenium angustifolium (Narrow-leaved Spleen- & 160 & \\
\hline 500 & Asplenium ebeneum (Ebony Spleenwort) & $\begin{array}{l}1.00 \\
1.00\end{array}$ & $\begin{array}{r}14.00 \\
8.00\end{array}$ \\
\hline 00 & Asplenium Filix-foemina (Lady Fern) & 1.00 & 8.00 \\
\hline 1500 & Asplenium thelypteroides (Silvery Spleenwort) & 1.00 & 8.00 \\
\hline 3000 & Asplenium trichomanes (Maidenhair Spleenwort) & 1.00 & 8.00 \\
\hline 1200 & Cystopteris bulbifera (Bladder Hern) & 1.00 & 8.00 \\
\hline 1000 & Cystopteris fragilis (Fragile Fern) & 1.00 & 8.00 \\
\hline 50 & Dicksonia punctilobula (Hayscented Fern) strong & & \\
\hline & clumps & 1.00 & 8.00 \\
\hline 2000 & Dicksonia punctilobula, S & 2. & 20.00 \\
\hline 1000 & Onoclea sensibilis (Sensitive Fern) & 1.00 & 8.00 \\
\hline 3000 & Onoclea struthiopteris (Ostrich Fern) & 1.25 & 10.00 \\
\hline & Ophioglossum vulgatum (Adder's-Tongue Fern) & 1.00 & 8.00 \\
\hline 2500 & Osmunda cinnanomea (Cinnamon Fern) & 1.25 & 10.00 \\
\hline 5000 & Osmunda Claytoniana & 1.25 & 10.00 \\
\hline 2000 & Osmunda regalis (Royal Fern) & 1.25 & 10.00 \\
\hline 1500 & Pliegopteris Dryopteris (Beech Fern) & 1.00 & 8.00 \\
\hline 150 & Phegopteris hexagonoptera & & 8.00 \\
\hline 1500 & Phegopteris polypodioides & 1.00 & 8.00 \\
\hline 2000 & Polypodium vulgare (Common Polypody) & 1.00 & 8.00 \\
\hline 500 & Pteris aquilina (Common Brake) & 1.00 & 8.00 \\
\hline 175 & Woodsia ilvensis (Rusty Woodsia) & 1.00 & 8.00 \\
\hline 250 & Woodsia obtusa (Obtuse-leaved Woodsia) & 1.00 & 8.00 \\
\hline & Woodwardia angustifolia (Chain Fern) & 1.50 & 12.00 \\
\hline 2000 & Woodwardia virginica & 1.00 & 8.00 \\
\hline
\end{tabular}




\section{HARDY PERENNIALS}

\begin{tabular}{|c|c|c|c|}
\hline & & Per 10 & Per 100 \\
\hline 500 & Actaea alba (White Baneberry) & $\$ 1.50$ & $\$ 12.00$ \\
\hline 500 & Actaearubra (Red Baneberry) & 1.75 & 15.00 \\
\hline 200 & Anthemis tinctoria & 1.00 & 8.00 \\
\hline 300 & Aquilegia alpina & 1.00 & 8.00 \\
\hline 2000 & Aquilegia canadensis & 1.00 & 8.00 \\
\hline 250 & Aquilegia vulgaris & 1.00 & 8.00 \\
\hline 300 & Aquilegia vulgaris alba & 1.00 & 8.00 \\
\hline 200 & Arabis Kelleri (a miniature rock Cress, new) & 2.00 & 15.00 \\
\hline 1300 & Arabis mollis & 1.00 & 8.00 \\
\hline 2500 & Arenaria caespitosa & 1.00 & 8.00 \\
\hline 1000 & Arisaema triphyllum & 1.00 & 8.00 \\
\hline 1000 & Asarum canadense (Wild Ginger) & 1.00 & 8.00 \\
\hline 2000 & Asclepias tuberosa (Butterfly Wéed) & 1.25 & 10.00 \\
\hline 1200 & Aster cordifolia & 1.00 & 8.00 \\
\hline 500 & Aster ericoides & 1.00 & 8.00 \\
\hline 1200 & Aster laevis & 1.00 & 8.00 \\
\hline 1500 & Aster linariifolius & 1.00 & 8.00 \\
\hline 650 & Aster multiflorus & 1.00 & 8.00 \\
\hline 1000 & Aster Novae-angliae & 1.00 & 8.00 \\
\hline 500 & Aster novi-belgii & 1.00 & 8.00 \\
\hline 200 & Aubrietia deltoides & 1.50 & 12.00 \\
\hline 400 & Baptisia tinctoria & 1.00 & 8.00 \\
\hline 1500 & Campanula rotundifolia & 1.25 & 10.00 \\
\hline 300 & Caulophyllum thalictroides (Blue Cohosh) & 1.00 & 8.00 \\
\hline 200 & Centaurea montana & 1.00 & 8.00 \\
\hline 400 & Chelone glabra & 1.00 & 8.00 \\
\hline 2000 & Cimicifuga racemosa & 1.00 & 8.00 \\
\hline 500 & Claytonia virginica & .75 & 6.00 \\
\hline 400 & Clintonia borealis & 1.00 & 8.00 \\
\hline 500 & Convallaria majalis (clumps 3 to 5 pips) & 1.25 & 10.00 \\
\hline 500 & Coptis trifolia & 1.00 & 8.00 \\
\hline 2000 & Cornus canadensis (clumps with earth) & 1.50 & 12.00 \\
\hline 500 & Dianthus barbatus & 1.00 & 8.00 \\
\hline 400 & Dianthus deltoides & 1.00 & 8.00 \\
\hline 300 & Dianthus plumarius & 1.00 & 8.00 \\
\hline 4000 & Dicentra Cucullaria & .75 & 6.00 \\
\hline 300 & Digitalis purpurea gloxiniaeflora & 1.00 & 8.00 \\
\hline 300 & Erysimum pulchellum & 1.00 & 8.00 \\
\hline 2000 & Erythronium albidum (large bulbs) & .75 & 6.00 \\
\hline 2000 & Erythronium Americanum (large bulbs) & .75 & 6.00 \\
\hline 1500 & Erythronium grandiflorum & .75 & 6.00 \\
\hline 800 & Eupatorium ageratoides & 1.00 & 8.00 \\
\hline 300 & Eupatorium perfoliatum & 1.00 & 8.00 \\
\hline 200 & Eupatorium purpureum & 1.00 & 8.00 \\
\hline 300 & Funkia Thomas Hogg & 1.00 & 8.00 \\
\hline 300 & Gaillardia grandiflora & 1.00 & 8.00 \\
\hline 2000 & Galax aphylla & 1.25 & 10.00 \\
\hline 2000 & Gaultheria procumbens (clumps with earth) & 1.50 & 12.00 \\
\hline 1500 & Gentiana Andrewsii & 1.00 & 8.00 \\
\hline 400 & Geranium maculatum & 1.00 & 8.00 \\
\hline 175 & Hemerocallis Gold Dust & 1.00 & 8.00 \\
\hline 2000 & Hepatica acutiloba (strong clumps) & 1.25 & 10.00 \\
\hline 1500 & Hepatica triloba & 1.00 & 8.00 \\
\hline 500 & Hibiscus mospheutos roseus & 1.00 & 8.00 \\
\hline 500 & Houstonia caerulea & .75 & 6.00 \\
\hline 400 & Hypoxis erecta & 1.00 & 8.00 \\
\hline 1000 & Iris cristata & 1.00 & 8.00 \\
\hline 150 & Iris hexagona & 1.00 & 8.00 \\
\hline 1400 & Iris pumila hybrida (mixed) & 1.25 & 10.00 \\
\hline 200 & Iris sibirica alba & 1.00 & 8.00 \\
\hline 1000 & Iris Snow Queen & 1.00 & 8.00 \\
\hline 1000 & Iris verna & 1.00 & 8.00 \\
\hline 1500 & Iris versicolor & 1.00 & 8.00 \\
\hline 250 & Liatris scariosa & 1.00 & 8.00 \\
\hline 3000 & Lilium canadense 1 st size ( $\$ 85$ per $M)$ & 1.25 & 10.00 \\
\hline 1500 & Lilium philadelphicum & 1.25 & 10.00 \\
\hline 2000 & Lilium superbum, 1 st size & 1.25 & 10.00 \\
\hline 1000 & Lobelia cardinalis & 1.00 & 8.00 \\
\hline 300 & Lupinus perennis & 1.00 & 8.00 \\
\hline 1200 & Lysimachia Nummularia & 1.00 & 8.00 \\
\hline 200 & Lythrum roseum superbum & 1.00 & 8.00 \\
\hline 7000 & Mertensia virginica, 1 st size & 1.00 & 8.00 \\
\hline 2000 & Mitchella repens (clumps with earth) & 1.25 & 10.00 \\
\hline 300 & Mitella diphylla & 1.00 & 8.00 \\
\hline 1200 & Myosotis palustris & 1.00 & 8.00 \\
\hline 500 & Oenothera fruticosa major & 1.00 & 8.00 \\
\hline 300 & Parnassia caroliniana & 1.00 & 8.00 \\
\hline 1600 & Phlox amoena & 1.00 & 8.00 \\
\hline 1500 & Phlox divaricata & 1.00 & 8.00 \\
\hline 8000 & Phlox subulata & 1.00 & 8.00 \\
\hline 2000 & Phlox subulata alba & 1.00 & 8.00 \\
\hline 2200 & Phlox subulata lilacina & 1.00 & 8.00 \\
\hline 1000 & Podophyllum peltatum & 1.00 & 8.00 \\
\hline
\end{tabular}




\begin{tabular}{|c|c|}
\hline Per 10 & Per 100 \\
\hline 1.00 & 8.00 \\
\hline 1.00 & 8.00 \\
\hline 1.25 & 10.00 \\
\hline 1.25 & 10.00 \\
\hline 1.25 & 10.00 \\
\hline 1.25 & 10.00 \\
\hline 1.25 & 10.00 \\
\hline .75 & 6.00 \\
\hline 1.00 & 8.00 \\
\hline 1.00 & 8.00 \\
\hline 1.00 & 8.00 \\
\hline 1.00 & 8.00 \\
\hline 1.00 & 8.00 \\
\hline 1.00 & 8.00 \\
\hline 1.00 & 8.00 \\
\hline 1.00 & 8.00 \\
\hline 2.50 & 20.00 \\
\hline 1.25 & 10.00 \\
\hline 1.00 & 8.00 \\
\hline 1.00 & 8.00 \\
\hline 1.00 & 8.00 \\
\hline 1.00 & 8.00 \\
\hline .75 & 6.00 \\
\hline 1.00 & 8.00 \\
\hline 1.00 & 8.00 \\
\hline 1.00 & 8.00 \\
\hline 1.00 & 8.00 \\
\hline 1.00 & 8.00 \\
\hline 1.00 & 8.00 \\
\hline 1.00 & 8.00 \\
\hline .65 & 5.00 \\
\hline 1.00 & 8.00 \\
\hline .75 & 6.00 \\
\hline .50 & 4.00 \\
\hline 1.00 & 8.00 \\
\hline 1.00 & 8.00 \\
\hline 1.00 & 8.00 \\
\hline 1.00 & 8.00 \\
\hline 1.00 & 8.00 \\
\hline 1.25 & 10.00 \\
\hline 1.00 & 8.00 \\
\hline 1.00 & 8.00 \\
\hline 1.00 & 8.00 \\
\hline 1.00 & 8.00 \\
\hline 1.00 & 8.00 \\
\hline .75 & 6.00 \\
\hline 1.00 & 8.00 \\
\hline 1.00 & 8.00 \\
\hline 1.00 & 8.00 \\
\hline 1.00 & 00 \\
\hline 1.00 & \\
\hline
\end{tabular}

\section{BOG PLANTS}

1000 Acorus calamus

1000 Calla palustris

2500 Caltha palustris

100 Dionaea muscipula (Venus Fly-trap)

500 Menyanthes trifoliata

700 Sarracenia flava

1000 Sarracenia rubra

800 Sarracenia purpurea

300 Scirpus atrovirens

1000 Vaccinium macrocarpon

$\begin{array}{rr}\text { Per 10 } & \text { Per 100 } \\ \$ 1.00 & \$ 8.00 \\ .75 & 6.00 \\ .75 & 6.00 \\ 1.50 & 15.00 \\ .75 & 6.00 \\ 1.25 & 10.00 \\ 1.25 & 10.00 \\ 1.00 & 8.00 \\ .75 & 6.00 \\ .75 & 6.00\end{array}$

\section{NATIVE ORCHIDS}

400 Calypso borealis

Per 10

Per 100

1500 Cypripedium acaule ( 1 to 2 crowns)

100 Cypripedium parviflorum ( 3 to 4 crowns)

145 Cypripedium parviflorum (5 to 8 crowns)

1.00 each

40.00

$\begin{array}{ll}7.50 & 15.00\end{array}$

200 Cypripedium pubescens ( 3 to 4 crowns)

$3.00 \quad 25.00$

125 Cypripedium pubescens ( 5 to 8 crowns)

300 Cypripedium spectabile ( 3 to 4 crowns)

100 Cypripedium spectabile ( 5 to 8 crowns)

500 Pogonia ophioglossoides 


\section{EVERGREEN SHRUBS}

Prices include balling and burlapping.

2100 Andromeda floribunda 12-15 in. spread

Per 10 Per 100

$\$ 17.50 \quad \$ 150.00$

$22.50 \quad 200.00$

(My Andromedas are heavily budded)

400 Calluna vulgaris clumps, (Scotch Heather) 6-8 in. B\&B

$6.00 \quad 50.00$

$8.50 \quad 70.00$

$\begin{array}{rr}8.50 & 100.00\end{array}$

200 Calluna vulgaris clumps, $8-10$ in. B\&B

200 Calluna vulgaris clumps, $10-12$ in. $B \& B$

1000 Daphne cneorum 8-10 in. B\&B

$7.00-60.00$

$9.00 \quad 80.00$

2000 Daphne cneorum 10-12 in. spread B\&B

110.00

$17.50 \quad 150.00$

Daphne cneorum 15 in. spread selected B\&B

85.00

$\begin{array}{rr}10.00 & 85.00 \\ 17.50 & 150.00\end{array}$

$8.50 \quad 75.00$

100 Ilex glabra 18-24 in.

2500 Kalmia latifolia $12-15$ in

3000 Kalmia latifolia 15-18 in.

1900 Kalmia latifolia $18-24$ in

1000 Kalmia latifolia $2 \mathrm{ft}$

500 Leiophyllum buxifolium 6-8 in.

200 Leiophyllum buxifolium 8-10 in

100 Leiophyllum buxifolium 10-12 in

100 Leiophyllum buxifolium 12-15 in.

1000 Leucothoe catesbaei $12-15$ in. clumps

650 Leucothoe catesbaei 15-18 in. clumps

1500 Pachysandra terminalis $2 \mathrm{yr}$.

$\begin{array}{ll}12.50 & 100.00 \\ 17.50 & 150.00\end{array}$

$17.50 \quad 150.00$

$20.00 \quad 175.00$

$\begin{array}{r}7.50 \quad 60.00 \\ \hline .00\end{array}$

$9.00 \quad 80.00$

10.00

12.50

$7.50 \quad 60.00$

$8.50 \quad 75.00$

$1.25 \quad 10.00$

All Rhododendrons in nursery grown grades have been grown from stock cut back to the ground; all new tops; bushy, with fine foliage.

$\begin{array}{rlrr}1650 & \text { Rhododendron carolinianum 12-15 in. } & \text { Per } 10 & \text { Per } 100 \\ 2200 & \text { Rhododendron carolinianum 15-18 in. } & \$ 12.50 & \$ 100.00 \\ 400 & \text { Rhododendron carolinianum 18 in. selected } & 16.50 & 145.00 \\ 100 & \text { Rhododendron catawbiense compacta 15-18 in. } & 22.50 & \\ 200 & \text { Rhododendron catawbiense compacta 18-24 in. } & 25.50 & \\ 1400 & \text { Rhododendron maximum 12-15 in. } & 10.00 & \\ 1850 & \text { Rhododendron maximum 15-18 in. } & 12.50 & 100.00 \\ 600 & \text { Rhododendron maximum 18-24 in. } & 16.00 & 140.00 \\ 500 & \text { Rhododendron maximum 18-24 in. heavy clumps } & 20.00 & 175.00\end{array}$

I will have at my nursery early in April the following Rhododendrons in freshly collected stock. Only plants of excellent quality will be sent out.

500 Rhododendron catawbiense $18-24$ in. $\quad 17.50 \quad 150.00$

500 Rhododendron catawbiense $2-3 \mathrm{ft} \quad 22.50 \quad 200.00$

1000 Rhododendron maximum 2-3 ft. $\quad 15.00 \quad 125.00$

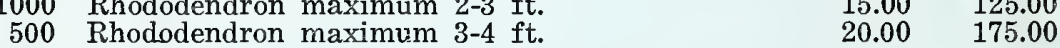

\section{DECIDUOUS SHRUBS}

The prices on Azaleas include balling and burlapping.

$\begin{array}{rr}\text { Per } 10 & \text { Per } 100 \\ 10.00 & 85.00 \\ 12.50 & 100.00 \\ 10.00 & 85.00 \\ 12.50 & 100.00 \\ 10.00 & 85.00 \\ 15.00 & 125.00 \\ 7.50 & 60.00 \\ 10.00 & 85.00 \\ 12.50 & 100.00 \\ 17.50 & 150.00 \\ & \\ 2.50 & 20.00 \\ 1.50 & 12.00 \\ 3.00 & 25.00 \\ 1.50 & 12.00 \\ 2.00 & \\ 1.50 & 12.00 \\ 1.00 & 8.00\end{array}$

20.00 Azalea calendulacea $12-15$ in.

1200 Azalea calendulacea 15-18 in.

800 Azalea canescens $12-15$ in.

200 Azalea canescens 15-18 in.

300 Azalea nudiflora $15-18$ in.

300 Azalea nudiflora $18-24$ in.

300 Azalea viscosa 15-18 in.

1000 Azalea viscosa $18-24$ in

1100 Azalea viscosa $2-3 \mathrm{ft}$.

650 Azalea viscosa $3 \mathrm{ft}$. selected

My Azaleas are all well budded.

600 Comptonia asplenifolia 12-15 in.

700 Clethra alnifolia 12-15 in.

200 Corylus americana $2 \frac{1}{2}-3 \mathrm{ft}$. strong

800 Diervilla trifida 18 in.

300 Myrica cerifica $12-15$ in.

100 Rosa lucida 12-18 in.

2000 Zanthorhiza apiifolia 6-8 in.

$1.00 \quad 8.00$

\section{Gillett's Fern and Flower Farm} KENNETH E. GILLETT, Proprietor 